\title{
Prevalence and associated factors of postpartum depression among immigrant women in Guangzhou, China
}

\author{
Ribo Xiong ${ }^{1,2}$ and Aiwen Deng ${ }^{1,2^{*}}$
}

\begin{abstract}
Background: Although there has been mounting research on postpartum depression (PPD), the impact of immigration on PPD has remained quite unexplored. The purpose of this study is to investigate the prevalence and associated factors of PPD among immigrant women living in Guangzhou at 6 weeks postpartum.

Methods: A cross-sectional study was conducted involving 1230 immigrant women in a tertiary hospital of Guangzhou from December 2016 to December 2017 at 6 weeks postpartum. The Chinese version of Edinburgh Postnatal Depression Scale and a structured questionnaire regarding associated factors were administered to all participants. Multivariate logistic regression was used to determine factors that were significantly associated with PPD.

Results: The prevalence of PPD among immigrant women at 6 weeks postpartum was 34.0\%. A multivariate logistic regression model identified significant obstetric and social factors as: living in Guangzhou for less than 2 years, insufficient family income, poor social support and marital relationship.

Conclusion: Prevalence of PPD among immigrant women from Guangzhou at 6 weeks postpartum is high. The development of PPD among immigrant women is associated with individual and social factors. There's an urgent need for healthcare providers to take a more active role in engaging immigrant women in their psychological needs.
\end{abstract}

Keywords: Immigrant, Postpartum depression, Prevalence, Associated factors, Social support

\section{Background}

Over the course of the last two decades or so, China has experienced the most extensive internal migration which has brought increased attention to the promotion of migrant-sensitive health policies and diseases prevention especially for migrant women and children. According to national statistics, up to 2015 , an estimated 277 million immigrants who were originally from the poor and rural areas in western and central inland provinces

\footnotetext{
* Correspondence: kara802@163.com

'Department of rehabilitation, Nanhai Hospital, Southern Medical University, Foshan, China

${ }^{2}$ General Practice Center, Nanhai Hospital, Southern Medical University, Foshan, China
}

\section{$\triangle B M C$}

C C The Author(s). 2020 Open Access This article is licensed under a Creative Commons Attribution 4.0 International License, which permits use, sharing, adaptation, distribution and reproduction in any medium or format, as long as you give appropriate credit to the original author(s) and the source, provide a link to the Creative Commons licence, and indicate if changes were made. The images or other third party material in this article are included in the article's Creative Commons licence, unless indicated otherwise in a credit line to the material. If material is not included in the article's Creative Commons licence and your intended use is not permitted by statutory regulation or exceeds the permitted use, you will need to obtain permission directly from the copyright holder. To view a copy of this licence, visit http://creativecommons.org/licenses/by/4.0/. The Creative Commons Public Domain Dedication waiver (http://creativecommons.org/publicdomain/zero/1.0/) applies to the data made available in this article, unless otherwise stated in a credit line to the data. such as Shanghai and Guangzhou for better opportunities and income [1]. Based on the definition of immigrants in the National Internal Migrant Dynamic Monitoring Survey, immigrants in our study refers to the individuals who do not have permanent resident certificate("Hukou") and should be living over a month in the residence. Due to the fact that the utility and allocation of public resources is based on household registration ("Hukou") policy, immigrants do not have the same rights and benefits as local registered residents in a variety of areas, such as health care, social services, offspring education and housing. In addition, immigrants 
have confronted challenges in a new host society, such as adjusting to a new language, unfamiliar laws, different norms for social interaction and lifestyle changes. How the acculturation affects immigrants' health status and the relationship between immigration process and the mental health of women continuously attract researcher's interest.

A growing number of studies suggest that immigrant women may be at increased risk of depression prenatally and postnatally [2-4]. A recent systematic review found that immigrant women are twice as likely to experience PPD as nonimmigrant women [5]. In a cross-sectional study conducted in Tokyo, Japan, migrant Chinese women had elevated depression symptoms postnatally than Japanese-born women [6]. Several studies conducted in Ontario, Canada have found migrant Chinese women had increased risk of PPD compared to their Canadian-born counterparts $[7,8]$. The existing literature mainly focused on Chinese migrants in Canada and other developed countries with significant disparities in culture and social environment. The status of PPD among Chinese internal migrants have remained quite unexplored. This is a significant limitation given the rapidly changing demographics in the south area of China where about 1 in 3 are identified as immigrants.

The principle aim of the present study is to examine the occurrence of PPD at 6 weeks after delivery among immigrant women. By exploring the biological, psychological and cultural factors, culturally appropriate and equitable health care services are provided to support immigrant mothers.

\section{Methods \\ Participants}

This is a cross-sectional study carried out from December 2016 to 2017 in a tertiary hospital of Guangzhou.The effect size was assumed to be 0.30 , referring the study of Gaskin, et al. [9] To achieve $80 \%$ power with an effect size of 0.5 and an alpha of 0.05 , a total sample size of 367 was needed. We recruited female immigrants at 6 weeks after childbirth 1) at childbearing age between 18 and 49;2) without "Hukou"(registered resident certificate) in Guangzhou and be living over a month in Guangzhou; 3) Chinese nationality; 4)providing informed consent. Women who had a history or family history of psychiatric diseases were excluded. Women who returned incomplete questionnaires were also excluded. A total of 1247 eligible women were invited to participate; 5 women refused to be enrolled in and 12 women returned incomplete questionnaires, resulting 1230 women. This study included 1230 immigrant women, allowing detection of significant differences with a power of 0.91 calculated by GPower software.

\section{Procedures and measures}

Investigators conducted face to face interview for data collection. The Chinese version of Edinburgh Postnatal Depression Scale (EPDS) was employed to assess PPD with a threshold of 10 . The sensitivity and specificity of Chinese version have been found to be 0.82 and 0.86 respectively which were comparable to the original scale [10].

Based on literature [11]and expert consultation, structured questionnaires [12] were administered to each women covering information including: 1) sociodemographic factors such as age, employment, labor contract, annual household income, years of residence; 2) obstetric factors such as parity, planned pregnancy, mode of delivery, pregnancy associated diseases, delivery associated diseases and fetus gender; 3) social factors such as perceived family income sufficiency, family socio-economic status, the ability to use local language, social support and marital relationship. Social support was ascertained by Likert scale with a range from 12 to 60 . The scale consists of three subscales: informational support (i.e, provision of advice, suggestions, information that the women could use), emotional support (i.e, provision of empathy, love, and caring), and household activity support. This scale demonstrated acceptable reliability and validity in a previous study [13].

The study was approved by the Research Ethics Board of Southern Medical University and written consent was sought from eligible participants.

\section{Data analysis}

The primary data was entered into Epidata3.0 before being exported to SPSS16.0. Chi-square test was used to assess the differences between two independent qualitative data and Student's $t$ test was used for quantitative data. Then the multivariate logistic regression was further employed to identify factors that were significantly associated with PPD. A $P$ value $<0.05$ was considered significant in the analysis.

\section{Results}

Out of 1230 immigrant women who were enrolled in the assessment, we have reported that 418 women screened positive for PPD with EPDS at a cut-off point of 10, thus resulting in a prevalence of $34.0 \%$.

Analysis of demographic profiles of immigrant women was shown in Table 1. Among all the factors concerning age, employment, labor contract, annual household income and years of residence, less recent immigrants showed significantly lower rates of PPD compared with recent immigrants.

Analysis of obstetric factors and probable PPD was shown in Table 2 . There were no significant differences 
Table 1 Demographic profiles of immigrant women with PPD

\begin{tabular}{|c|c|c|c|c|c|c|}
\hline \multirow[t]{2}{*}{ Factors } & \multicolumn{2}{|c|}{ EPDS < $10(812)$} & \multicolumn{2}{|c|}{ EPDS $\geq 10(418)$} & \multirow[t]{2}{*}{$x^{2}$} & \multirow[t]{2}{*}{$P$} \\
\hline & $n$ & $\%$ & $n$ & $\%$ & & \\
\hline Age & & & & & 1.010 & 0.799 \\
\hline$\leq 24$ & 123 & 15.15 & 67 & 16.03 & & \\
\hline $25 \sim 29$ & 504 & 62.07 & 260 & 62.20 & & \\
\hline $30 \sim 34$ & 112 & 13.79 & 60 & 14.35 & & \\
\hline$\geq 35$ & 73 & 8.99 & 31 & 7.42 & & \\
\hline Employment & & & & & 0.516 & 0.259 \\
\hline Yes & 553 & 68.10 & 293 & 70.10 & & \\
\hline No & 259 & 31.90 & 125 & 29.90 & & \\
\hline Labor contract & & & & & 2.275 & 0.810 \\
\hline Unfixed term & 298 & 36.70 & 160 & 38.28 & & \\
\hline Fixed term & 275 & 33.87 & 142 & 33.97 & & \\
\hline One-time task or a probation period & 138 & 17.00 & 69 & 16.50 & & \\
\hline Unsign labor contract & 79 & 9.73 & 35 & 8.37 & & \\
\hline Unknown & 14 & 1.72 & 10 & 2.39 & & \\
\hline Else & 8 & 0.98 & 2 & 0.48 & & \\
\hline Annual household income & & & & & 5.164 & 0.160 \\
\hline 60,000 99999RMB & 210 & 25.86 & 130 & 31.10 & & \\
\hline 100,000 139999RMB & 389 & 47.90 & 187 & 44.74 & & \\
\hline $140,000 \sim 179,999$ RMB & 131 & 16.13 & 55 & 13.16 & & \\
\hline$\geq 180000 \mathrm{RMB}$ & 82 & 10.10 & 46 & 11.00 & & \\
\hline Years of residence & & & & & 74.383 & 0.000 \\
\hline$\leq 2$ years & 190 & 23.40 & 188 & 44.98 & & \\
\hline $2 \sim 5$ years & 324 & 39.90 & 155 & 37.08 & & \\
\hline $6-10$ years & 298 & 36.70 & 75 & 17.94 & & \\
\hline
\end{tabular}

between immigrant women with PPD and those without regarding obstetrics factors.

Table 3 represented social factors and probable PPD. Insufficient family income, lower social support and poor marital relationship were significantly associated with the development of PPD among immigrant women.

Table 4 showed the associated factors of PPD in immigrant women. The strongest factor was lower social support. Immigrant women with lower social support was 3.287 times more likely to develop PPD. In relation to perceived family income, immigrant women who reported insufficiency of family income were at greater risk of PPD $(\mathrm{OR}=2.413,95 \% \mathrm{CI}: 1.991 \sim 3.134)$. Similarly, poor marital relationship was identified as a risk factor $(\mathrm{OR}=2.317$, 95\%CI: $1.917 \sim 2.739$ ). An increased risk was also observed in immigrant women with less than 2 years of residence in Guangzhou (OR $=3.018,95 \%$ CI: $2.589 \sim 3.422)$.

\section{Discussion}

Guangzhou, where this study was conducted, is one of the largest migrant-concentrated metropolis in China.
With nearly $50 \%$ of it's total population being migrated and $50 \%$ of it's migrants being female, Guangzhou provided a natural environment where the maladjustment such as depression and anxiety can be observed among immigrant women [14]. The prevalence rate of PPD was as high as $34.0 \%$. Likewise, a Japanese study of new mothers found that $54.5 \%$ of migrant Chinese women had elevated levels of postpartum depressive symptoms as indicated by scores on EPDS [6]. Several other Canadian studies similarly found that the risks for PPD in immigrant Chinese women were significantly higher than that of native-born women [2-4]. In another sample of immigrant women in Taiwan, higher prevalence of PPD (41.1\% vs. $8.4 \%)$ was also identified [15]. However, studies that compare US-born Hispanic mothers with their foreign-born counterparts suggested a lower rate in the latter [16]. In another epidemiological study, no difference of PPD rates was observed among foreign-born mothers and US-born mothers, but not among Whites or Asian/Pacific Islanders [17]. The variation of the prevalence highlights the complex interplay of social, 
Table 2 Obstetric characteristics of immigrant women with PPD

\begin{tabular}{|c|c|c|c|c|c|c|}
\hline \multirow[t]{2}{*}{ Factors } & \multicolumn{2}{|c|}{ EPDS < 10 (812) } & \multicolumn{2}{|c|}{ EPDS $\geq 10(418)$} & \multirow[t]{2}{*}{$x^{2}$} & \multirow[t]{2}{*}{$P$} \\
\hline & $n$ & $\%$ & $n$ & $\%$ & & \\
\hline Parity & & & & & 0.159 & 0.706 \\
\hline 1 & 291 & 36.21 & 145 & 34.69 & & \\
\hline$\geq 2$ & 521 & 64.16 & 273 & 65.31 & & \\
\hline Pregnancy planning & & & & & 1.889 & 0.184 \\
\hline Unplanned & 423 & 52.09 & 235 & 56.22 & & \\
\hline Planned & 389 & 47.91 & 183 & 43.78 & & \\
\hline Mode of delivery & & & & & 1.736 & 0.207 \\
\hline Vaginal & 624 & 76.85 & 307 & 73.44 & & \\
\hline Caesarean & 188 & 23.15 & 111 & 26.56 & & \\
\hline Pregnancy related disease & & & & & 0.266 & 0.632 \\
\hline Yes & 217 & 26.72 & 106 & 23.36 & & \\
\hline No & 595 & 73.28 & 312 & 74.64 & & \\
\hline Delivery related disease & & & & & 0.723 & 0.434 \\
\hline Yes & 108 & 13.30 & 63 & 15.07 & & \\
\hline No & 704 & 86.70 & 355 & 84.92 & & \\
\hline Fetus gender & & & & & 0.724 & 0.399 \\
\hline Male & 428 & 52.71 & 231 & 55.26 & & \\
\hline Female & 384 & 47.29 & 187 & 44.74 & & \\
\hline
\end{tabular}

Table 3 Social profiles of immigrant women with PPD

\begin{tabular}{|c|c|c|c|c|c|c|}
\hline \multirow[t]{2}{*}{ Factors } & \multicolumn{2}{|c|}{$\underline{\text { EPDS }<10(812)}$} & \multicolumn{2}{|c|}{ EPDS $\geq 10(418)$} & \multirow[t]{2}{*}{$x^{2} / t$} & \multirow[t]{2}{*}{$P$} \\
\hline & $\mathrm{n} \%$ & & $n$ & $\%$ & & \\
\hline \multicolumn{5}{|l|}{ Perceived family income sufficiency } & 95.088 & 0.000 \\
\hline Not enough & 259 & 31.90 & 251 & 60.05 & & \\
\hline Just enough & 408 & 50.25 & 139 & 33.25 & & \\
\hline More than enough & 145 & 17.85 & 28 & 6.70 & & \\
\hline \multicolumn{5}{|l|}{ Family socio-economic status } & 3.102 & 0.212 \\
\hline Low & 263 & 32.39 & 147 & 35.17 & & \\
\hline Middle & 514 & 63.30 & 246 & 58.85 & & \\
\hline High & 35 & 4.31 & 25 & 5.98 & & \\
\hline \multicolumn{5}{|l|}{ The ability to use local language } & 2.878 & 0.237 \\
\hline Poor & 468 & 57.64 & 259 & 61.96 & & \\
\hline Moderate & 240 & 29.56 & 117 & 27.99 & & \\
\hline Satisfying & 104 & 12.80 & 42 & 10.05 & & \\
\hline Social support & \multicolumn{2}{|c|}{$38.42 \pm 9.21$} & \multicolumn{2}{|c|}{$31.08 \pm 10.24$} & -15.655 & 0.000 \\
\hline \multicolumn{5}{|l|}{ Marital relationship } & 104.2 & 0.000 \\
\hline Poor & 178 & 21.92 & 210 & 50.24 & & \\
\hline Moderate & 245 & 30.17 & 92 & 22.01 & & \\
\hline Satisfying & 389 & 47.91 & 116 & 27.75 & & \\
\hline
\end{tabular}


Table 4 Multivariate logistic regression analysis of impact factor of PPD in immigrant women

\begin{tabular}{|c|c|c|c|c|c|c|}
\hline Covariates & $B$ & S.E & Wald & $P$ & $O R$ & $95 \% \mathrm{Cl}$ \\
\hline Age & 0.087 & 0.151 & 14.219 & 0.658 & 1.041 & $0.455 \sim 1.328$ \\
\hline Employment & 0.973 & 0.824 & 28.982 & 0.471 & 2.634 & $2.006 \sim 3.140$ \\
\hline Labor contract & 0.056 & 0.138 & 17.231 & 1.025 & 3.177 & $2.216 \sim 3.841$ \\
\hline Annual household income & 0.991 & 1.472 & 15.365 & 3.007 & 1.945 & $1.090 \sim 3.149$ \\
\hline \multicolumn{7}{|l|}{ Years of residence } \\
\hline$\leq 2$ years & 1.763 & 0.381 & 33.105 & 0.000 & 3.018 & $2.589 \sim 3.422$ \\
\hline $2 \sim 5$ years & -0.897 & 0.153 & 27.314 & 0.094 & 1.229 & $0.877 \sim 1.681$ \\
\hline $6-10$ years & -0.776 & 0.169 & 19.233 & 1.241 & 1.586 & $1.040 \sim 1.989$ \\
\hline Parity & 1.263 & 4.287 & 47.116 & 0.987 & 2.019 & $1.114 \sim 2.840$ \\
\hline Pregnancy planning & 0.016 & 0.125 & 3.684 & 0.065 & 1.217 & $0.830 \sim 1.607$ \\
\hline Mode of delivery & -0.339 & 0.700 & 56.139 & 1.162 & 2.311 & $2.059 \sim 3.327$ \\
\hline Pregnancy related disease & 0.159 & 0.230 & 9.116 & 3.670 & 1.652 & $1.162 \sim 2.080$ \\
\hline Delivery related disease & 0.094 & 0.113 & 14.214 & 0.951 & 1.643 & $0.744 \sim 2.016$ \\
\hline Fetus gender & 0.052 & 0.136 & 10.050 & 0.083 & 2.097 & $1.339 \sim 3.017$ \\
\hline \multicolumn{7}{|l|}{ Perceived family income sufficiency } \\
\hline Not enough & 0.631 & 1.220 & 15.687 & 0.000 & 2.413 & $1.991 \sim 3.134$ \\
\hline Just enough & 0.248 & 0.746 & 11.501 & 0.077 & 1.359 & $0.423 \sim 2.982$ \\
\hline More than enough & - & - & - & - & - & - \\
\hline Family socio-economic status & -0.467 & 0.189 & 17.564 & 0.136 & 1.153 & $0.671 \sim 1.622$ \\
\hline The ability to use local language & -0.990 & 0.231 & 56.071 & 0.068 & 2.376 & $2.119 \sim 3.075$ \\
\hline Social support & 1.842 & 0.126 & 32.005 & 0.000 & 3.287 & $2.903 \sim 3.720$ \\
\hline \multicolumn{7}{|l|}{ Marital relationship } \\
\hline Poor & 0.021 & 0.108 & 44.168 & 0.000 & 2.317 & $1.917 \sim 2.739$ \\
\hline Moderate & 0.069 & 0.504 & 34.180 & 0.106 & 1.288 & $0.636 \sim 1.751$ \\
\hline Satisfying & - & - & - & - & - & - \\
\hline
\end{tabular}

Adjusted for all the above variates

cultural and systemic factors in the development of PPD. Higher rates of PPD in this study may be explained by differences across time point, social and cultural norms. First, women were screened for PPD at 6 weeks postpartum. Generally speaking, the rates obtained closer to delivery are more likely to be higher than those conducted later because of the care and support from their families after childbirth [18]. Second, China's internal migrants encountered "Hukou"-based social exclusion rather than ethnicity-based social exclusion experienced by international migrants. They are also more likely to face barriers such as knowledge regarding access to services and differences in language and lifestyle. We hypothesized social exclusion resulting from the "Hukou" policy as well as other economic and cultural factors are associated with adverse mental health consequences of immigrants. Further study is needed to explore the relationship between social exclusion and PPD among immigrant women.

This is one of the few studies examining length of time in the host society as an associated factor of PPD at 6 weeks. Having lived in Guangzhou for less than 2 years was significantly associated with PPD at 6 weeks. Similarly, immigrant women within previous 2 years in Canada were at increased risk for PPD at 6 weeks postpartum [11]. This might be attributed to the difficulties in adaptation to the host society and absence of usual support that would have been received from their family of origin. Furthermore, newcomers may have practical barriers such as equitable access to postnatal care, language difficulties and fear of stigma. This result has high policy relevance for local government to promote social integration for immigrants.

We also found a strong association between perceived family income insufficiency and PPD at 6 weeks postpartum. Our finding of perceived family income as an important factor of PPD is consistent with previous reviews focused on the overall postpartum population and a recent research on determinants of PPD among Chinese immigrants to high-income countries $[5,11,19]$. It's noteworthy that immigrant women were at higher risk of PPD even after accounting for insufficient family 
income, social support and domestic making power [20]. One of the possible explannation could be that immigrant mothers are mostly financially dependent on their spouses. The decreased status in the family as well as the stress of adapting to a new society could lead to a sense of helpless. This in turn could be related to less domestic making power which has been demonstrated to be a predictor of PPD in a research [20]. Future study could include women from different cultural backgrounds to better understand the relationship between family income and PPD among immigrant mothers.

Low social support was coherently associated with the development of PPD and has greater implications for immigrant women with limited or no social support [11, 18]. For the immigrant women in our study, those who received lower social support were up to three times more likely to develop PPD. It's a cultural imperative for Chinese women to observe a special tradition after childbirth called "doing the month". This tradition includes a set of practices involving prescribed diet, clothing and other cautions that must be followed during the first month after delivery. Numerous studies have revealed "doing the month" have positive influence on the recovery of the mother and successful adaptation to maternal role [21, 22]. Not following this ritual could lead to postpartum difficulties. However, because of comparatively lower income, unstable housing and absence of caregivers during the period of "doing the month", the psychosomatic recovery of immigrant mothers are negatively affected. The impact of low social support on PPD highlights the need to improve social welfare system for immigrants.

Poor marital relationship played an important role in the occurrence of PPD as observed in previous studies [5]. A research revealed feeling of isolation and loneliness resulting from poor marital relationship may lead to depressive symptoms among Thai women living in Australia [18]. Since most immigrant mothers depend on their husband financially, it's possible that financial challenges or lack of social networks are associated with increased prevalence of PPD. On the other hand, in Chinese culture, poor marital relationship or even domestic violence is regarded as stigma. Immigrant women may be reluctant to seek help in order to maintain the idealization of motherhood as a joyful period.

The results of our study should be considered with a few caveats. Firstly, a cross-sectional study did not allow us to establish a causal relationship. Secondly, EPDS was employed as screening tool to define PPD instead of a clinician-administered structured diagnostic interview. Thirdly, the recruitment of women from one of the districts in Guangzhou would limit the generalizability of the findings. Fourthly, report bias may exist regarding parity and sufficiency of family income. Given the sensibility of the topics, migrants might feel shy to reveal or decline because of social desirability bias.

\section{Conclusions}

The prevalence rate of PPD among immigrant women was high in Guangzhou, China. Recent immigrants, insufficient family income, low social support and poor marital relationship are risk factors that can contribute to PPD over the 6 weeks following childbirth. There's an urgent need for healthcare providers to take a more active role in engaging immigrant women in their psychological needs. Social support and culturally tailored programs should also be developed to prevent or alleviate depressive symptoms.

Abbreviations

EPDS: Edinburgh Postnatal Depression Scale; PPD: postpartum depression

\section{Acknowledgements}

We are grateful to the immigrant women who took their time to participate in the study.

\section{Authors' contributions}

AWD and RBX conceived the study, the methodology was developed by RBX, RBX carried out the interviews. AWD and RBX analyzed the interviews. RBX wrote the first draft of the manuscript and AWD edited the manuscript. All authors read and approved the final manuscript.

\section{Funding}

Not applicable.

\section{Availability of data and materials}

The datasets used and/or analyzed during the current study are available from the corresponding author on reasonable request.

\section{Ethics approval and consent to participate}

Ethical approval was obtained from the Research Ethics Board of Southern Medical University on January 12th, 2014. Participants were provided with a participant information sheet and asked to sign a consent form prior to the interviews being undertaken.

Consent for publication

Not applicable.

Competing interests

The authors declare that they have no competing interests.

Received: 2 April 2019 Accepted: 14 April 2020

Published online: 25 April 2020

\section{References}

1. The national health and family planning commission of the floating population family planning service management: China floating populationdevelopment report 2014. http://www.nhfpc.gov.cn/zhuzhan/ zxfb/201411/ce4a10d761944b2c85bfb99374fed040.shtml. Accessed 12 Aug 2018.

2. Danaci AE, Dinç G, Deveci A, Sen FS, Içelli I. Postnatal depression in Turkey: epidemiological and cultural aspects. Soc Psychiatry Psychiatr Epidemiol. 2002;37(3):125-9.

3. Dankner R, Goldberg RP, Fisch RZ, Crum RM. Cultural elements of postpartum depression. A study of 327 Jewish Jerusalem women. J Reprod Med. 2000;45:97-104.

4. Glasser S, Barell V, Shoham A, Ziv A, Boyko V, Lusky A, Hart S. Prospective study of postpartumdepression in an Israeli cohort: prevalence, incidence, and demographic riskfactors. J Psychosom Obstet Gynaecol. 1998;19(3):15564 
5. Falah-Hassani K, Shiri R, Vigod S, Dennis CL. Prevalence of postpartum depression among immigrant women: a systematic review and metaanalysis. J Psychiatr Res. 2015;70:67-82.

6. Jin Q, Mori E, Sakajo A. Risk factors, cross-cultural stressors and postpartum depression among immigrant Chinese women in Japan. Int J Nurs Pract. 2016;22(Suppl 1):38-47.

7. Zelkowitz P, Schinazi J, Katofsky L, Saucier JF, Valenzuela M, Westreich R, Dayan J. Factorsassociated with depression in pregnantimmigrantwomen. Transcult Psychiatry. 2004;41(4):445-64.

8. Zelkowitz P, Saucier JF, Wang T, Katofsky L, Valenzuela M, Westreich R. Stability and change in depressive symptoms from pregnancy to two months postpartum in childbearing immigrant women. Arch Womens Ment Health. 2008;11(1):1-11.

9. Gaskin CJ, Happell B. Power, effects, confidence, and significance: an investigation of statistical practices in nursing research. Int J Nurs Stud. 2014;51:795-806

10. Lee DT, Yip SK, Chiu HF, Leung TY, Chan KP, Chau IO, Leung HC, Chung TK. Detecting postnatal depression in Chinese women. Validation ofthe Chinese version of the Edinburg Postnatal Depression Scale. Br J Psychiatry. 1998; 172:433-7.

11. Ganann R, Sword W, Thabane L, Newbold B, Black M. Predictors of postpartum depression among immigrant women in the year after childbirth. J Women's Health (Larchmt). 2016;25(2):155-65.

12. Deng AW, Xiong RB, Jiang TT, Luo YP, Chen WZ. Prevalence and risk factors of postpartum depression in a population-based sample of women in Tangxia community, Guangzhou. Asian Pac J Trop Med. 2014;7(3):244-9.

13. Chen TL, Tai CJ, Chu YR, Han KC, Lin KC, Chien LY. Cultural factors and social support related to breastfeeding among immigrant mothers in Taipei City, Taiwan. J Hum Lact. 2011;27(1):41-8.

14. Guangdong statistical information: The data of the Sixth National Population Census of Guangzhou city. http://www.gzstats.gov.cn/pchb/ rkpc6/. Accessed 4 Jan 2020.

15. Chen HH, Hwang FM, Tai CJ, Chien LY. The interrelationships among acculturation, social support, and postpartum depression symptoms among marriage-based immigrant women in Taiwan: a cohortstudy. J Immigr Minor Health. 2013;15(1):17-23.

16. Davila M, McFall S, Cheng D. Acculturation and depressive symptoms among pregnant and postpartum Latinas. Matern Child Health J. 2009;13(3): $318-25$.

17. Huang ZJ, Wong FY, Ronzio CR, Yu SM. Depressive symptom-tology and mental health help-seeking patterns of US-and foreign- born mothers Matern Child Health J. 2007;11(3):257-67.

18. Ramakrishna S, Cooklin AR, Leach LS. Comorbid anxiety and depression: a community-based study examining symptomology and correlates during the postpartum period. J Reprod Infant Psychol. 2019;20:1-12.

19. Chen J, Cross WM, Plummer V, Lam L, Tang S. A systematic review of prevalence and risk factors of postpartum depression in Chinese immigrant women. Women Birth. 2018:S1871-5192(18):30210-5.

20. Chien LY, Tai CJ, Yeh MC. Domestic decision-making power, social support, and postpartum depression symptoms among immigrant and native women in Taiwan. Nurs Res. 2012;61(2):103-10.

21. Klainin P, Arthur DG. Postpartum depression in Asian cultures: a literature review. Int J Nurs Stud. 2009;46(10):1355-73.

22. Liu S, Yan Y, Gao X, Xiang S, Sha T, Zeng G, He Q. Risk factors for postpartum depression among Chinese women: path model analysis. BMC Pregnancy Childbirth. 2017;17(1):133.

\section{Publisher's Note}

Springer Nature remains neutral with regard to jurisdictional claims in published maps and institutional affiliations.

Ready to submit your research? Choose BMC and benefit from:

- fast, convenient online submission

- thorough peer review by experienced researchers in your field

- rapid publication on acceptance

- support for research data, including large and complex data types

- gold Open Access which fosters wider collaboration and increased citations

- maximum visibility for your research: over $100 \mathrm{M}$ website views per year

At BMC, research is always in progress.

Learn more biomedcentral.com/submissions 